\title{
On the Melting Process of Solids
}

\author{
U. Köbler ${ }^{1, *}$, V.Yu. Bodryakov ${ }^{2}$ \\ ${ }^{1}$ Research Centre Jülich, Institute PGI, 52425 Jülich, Germany \\ ${ }^{2}$ Ural State Pedagogical University, Yekaterinburg, 620119 Russia \\ E-mail:*u.koebler@fz-juelich.de
}

Received 26 February 2015, Revised 08 July 2015, Accepted 09 July 2015

\begin{abstract}
High temperature heat capacity data of the same solid reported by different authors can differ from each other by much more than can reasonably be attributed to the experimental errors, and seem to have a systematic origin. In this communication it will be shown that each individual data set can adequately be described by a "critical" power function of type $\sim\left(T_{m}-T\right)^{\alpha}$ plus an absolute constant $\left(T_{m}=\right.$ melting temperature). Commonly the critical power function holds for all heat capacity data beyond the atomistic Dulong-Petit (D-P) limit. Within the large critical range crossover phenomena between different power functions with different exponents $\alpha$ can additionally occur. For the asymptotic power functions $\left(T \rightarrow T_{m}\right)$ exponents near to the rational numbers of $\alpha=2 / 3,1$ and $3 / 2$ are identified. For the non asymptotic power functions the identified exponents are $\alpha=0$ (logarithmic divergence), $1 / 2$ and 2. Quite generally, a large validity range of the critical power function indicates that the heat capacity is not of atomistic origin but has to be attributed to a field of freely propagating bosons. This view is in analogy to the main issue of Renormalization Group (RG) theory that the dynamics in the vicinity of the magnetic ordering transition is not due to exchange interactions between spins but due to a boson guiding field. The postulated bosons at melting transition are not specified as yet but they are evidently excitations of the continuous solid with energies of much larger than the atomistic excitations (phonons). The floating heat capacity near $T_{m}$ can be explained by a mean free path of the bosons that is of the order of the linear dimension of the sample. The heat capacity of the field then depends on size, shape and surface quality of the sample. It therefore appears not possible to define an intrinsic behavior.
\end{abstract}

\section{Keywords: High temperature heat capacity; universality; critical dynamics.}

\section{Introduction}

Melting of solids is a phase transition of first order. This does not mean that there is no critical dynamics observable in the vicinity of $T_{m}$ [1]. In fact, it proves that heat capacity data can adequately be described by a critical power function of type $\sim\left(T_{m}-T\right)^{\alpha}$ plus an absolute constant. The absolute constant gives the heat capacity at $T_{m}$, the exponent $\alpha$ is always positive. With the exception of solid neon [2] melting temperatures are much higher than conforms to the interatomic forces (phonons), as they are well known from inelastic neutron scattering studies [3]. In other words $T_{m}$ is considerably larger than Debye temperature $\Theta_{D}$. Another puzzling experimental observation is that the heat capacity at melting temperature is much larger than the atomistic Dulong-Petit (D-P) limit of $3 R$ ( $R=$ gas constant). Larger heat capacities than D-P limit are difficult to explain by atomistic concepts [4]. It is therefore suggestive to conclude that there must be unknown energy degrees of freedom in addition to phonons that stabilize cohesion of the solid up to a surprisingly high melting temperature. The two typical energies can be characterized by $k_{B} \Theta_{D}$ and $k_{B} T_{m}$. As additional energy degrees of freedom we identify bosons of the continuous solid. Note that the characteristic symmetry of the continuous solid is continuous translational invariance. This symmetry acts as generator of ballistic propagating bosons. The bosons in question stabilize cohesion of the solid similar to the binding between quarks by gluons.

In contrast to the atomistic models the observed heat capacity at constant (or no) pressure, $c_{p}$, does not saturate at D-P limit but increases further up to melting temperature $T_{m}$.
For most solids the $c_{p}(T)$ data beyond D-P limit follow a perfectly linear function of temperature up to $T_{m}$ [5]. In addition to $\alpha=1, \alpha \sim 2 / 3,1 / 2$ and 1.5 also can be identified. Power functions of temperature that hold up to a large distance from critical temperature are the typical indication of the dynamics of a field of freely propagating bosons [6]. The large validity range of the critical power function results because the dispersion relations of freely propagating bosons are simple power functions of wave vector. Note that the critical power functions predicted by atomistic models hold asymptotically at critical point only. The functional change of $c_{P}(T)$ from a saturation like behavior for $c_{p}<3 R$ to a further increasing (concave up) behavior for $c_{p}>3 R$ has the character of a crossover event [6]. A crossover commonly indicates a change of the relevant excitation spectrum [7]. In agreement with the principles of Renormalization Group (RG) theory it can be assumed that the crossover at $c_{p} \sim 3 R$ is from the excitation spectrum of the phonons to the excitation spectrum of the boson field $[8,9]$. The two thermal reservoirs get alternatively activated (or relevant). Since excitation spectra can be classified by symmetries a crossover means a change in dynamic symmetry. The generic symmetries are the periodic translational symmetry of the discrete lattice and the continuous translational symmetry of the macroscopic or infinite solid. Note that symmetries and dispersion relations of phonons and field bosons are determined by their propagation modes. Phonons propagate from atom to atom while bosons propagate ballistic, independent of atomistic structures. Universality, in the sense of a finite critical range, is the typical thermodynamic behavior of a field of freely 
propagating bosons. It is evident that in temperature regions where the boson field is the relevant excitation spectrum the observed heat capacity is that of the boson field.

Published high temperature $c_{p}(T)$ data of the same material can differ appreciably from experiment to experiment [10]. The aim of this communication is to propose an explanation for this hitherto unexplained phenomenon. It turns out that universal power functions of type $\sim\left(T_{m}-T\right)^{\alpha}$ can be fitted to each individual data set, though with different exponents $\alpha$ and different absolute constants. For the temperature range with atomistic dynamics $\left(c_{p}<D-P\right.$ limit) published $c_{p}$ data for the same material agree considerably better [10]. As a conclusion, there must be an unknown parameter that has to be made responsible for the floating heat capacity data with $c_{p}>3 R$. For the proposed solution of this problem we assume that the mean free path of the bosons near $T_{m}$ has a comparable length as the linear dimension of the sample. The large mean free path implies a new mesoscopic length scale to the dynamics. Scattering of the bosons at grain boundaries or at the inner surfaces of the sample can modify the density of states of the field and therefore can change the universality class. The heat capacity therefore can depend on size, shape and surface quality of the sample and on whether the sample is a single crystal or a polycrystal. Unfortunately, these experimental details were considered as unimportant and were rarely communicated explicitly. In fact, from an atomistic point of view the interatomic distance is the only length scale. As a consequence, for atomistic models the shape of the sample should be unimportant. Furthermore, melting temperatures reported for the same material scatter occasionally by more than can reasonably be attributed to the experimental uncertainties. For instance, the reported melting temperatures for niobium scatter within the broad window of $T_{m} \sim 2688-2741 \mathrm{~K}$. This is consistent with the view that not only the critical dynamics but the actual value of the melting temperature also is determined by the boson field. This is as in magnetism where it is customary to call the boson driven ordering temperature a stable fixed point (SFP) in order to distinguish it from the ordering temperature estimated by atomistic models on the basis of the exchange interactions between spins.

The supposed bosons and their sources are, however, completely unexplored. This applies to most bosons at other types of order-disorder phase transitions as well. Only for the magnetic ordering transition it seems to be clear that the field bosons essentially are magnetic dipole radiation emitted upon precession of the ordered magnetic moments [7]. As long as the field quanta are unknown, the basis for the formulation of a realistic Lagrangian of the field is not yet given. It is only clear that the field bosons near $T_{m}$ must have larger excitation energies than phonons. Excitations with larger energies than phonons could be deformation modes of the surface of the atoms. These modes get activated only when all phonons are activated. The surface regions in question are involved in the chemical bond. In other words, atoms can no longer be considered as hard spheres but seem to possess internal energy degrees of freedom. Alternatively, many-body excitations have to be considered as well. However, using inelastic neutron scattering, no higher excitation energies than the well known single phonon processes have been reported [3]. This shows that the harmonic approximation is not so bad.

As a conclusion, melting of solids has to be explained by field theories rather than by atomistic concepts. Basically there is a twofold problem to be solved by field theories, first, the sources of the bosons have to be found out and, second, the bosons themselves have to be identified. Only for the electromagnetic radiation field the two problems have been solved exactly by quantum electrodynamics. For the Debye boson field it is clear that the bosons are sound waves. However it is completely unclear how sound waves are spontaneously generated in thermal equilibrium with an external temperature reservoir. Fortunately, if the field is the relevant thermal reservoir it suffices to consider the energy degrees of freedom of the field exclusively. The (weak) coupling between field particles and field sources needs not to be known if the field can be assumed to be in thermal equilibrium.

High temperature heat capacity data were frequently interpreted in terms of anharmonic effects in the lattice vibrations [11]. Those effects modify the phonon dispersion relations very little. In particular, anharmonic inter-atomic interactions do not create further states in addition to $3 \mathrm{~N}$ ( $N=$ Avogadro number). Consequently, the heat capacity does not exceed D-P limit of $3 R$. Since the observed phonon energies are too small to explain the high melting temperatures anharmonic effects seem not to be essential for the melting process.

Thermal generation of vacancies has occasionally been discussed as a mechanism contributing to the increasing lattice expansion and heat capacity on approaching melting point. However, the fitted activation energies for vacancy formation are much higher than the melting temperature $[12,13]$. In those cases where the high temperature heat capacity exhibits perfect linear temperature dependence the activation energy for vacancy formation, formally, is infinite. From the weak non linearity in the lattice parameter of lead an activation energy for the generation of vacancies of $\sim 0.5$ $\mathrm{eV}(\approx 5800 \mathrm{~K})$ has been deduced [14]. As a consequence, thermal generation of vacancies is a weak process at the temperatures considered here.

Universality, in the definition of a finite validity range of the critical power functions, is best known for the critical magnetic dynamics [15] and for the vapor-liquid transition $[16,17]$. From the surprising similarity of the critical exponents at vapor-liquid transition and at the magnetic ordering transition one can conclude that the responsible bosons have similar dispersion relations and densities of state. In other words, physically very different boson fields such as the Debye boson field and the electromagnetic radiation field can have identical thermodynamic properties if the field quanta have identical dispersion relations. For the massless Debye bosons and photons the dispersion relation is a linear function of wave vector. The energy densities of the two fields are $\sim T^{4}$.

Bosons are difficult to observe directly. In particular, massless bosons are invisible to neutrons. The only thermodynamic observable for a boson field is its heat capacity. Only in ferromagnets experimental information on the dispersion relations of the bosons could be obtained from experiments on resonating boson states in confined geometries [18]. Relevance of the boson field means that the passive spin system receives its thermodynamic behavior from the field. Thermal decrease of the magnetic order parameter is controlled by the heat capacity of the field [7].

\section{Analysis of Experimental Data}

For simplicity we restrict discussion to the metallic monoatomic solids $\mathrm{V}, \mathrm{Nb}, \mathrm{Ta}$, Mo and $\mathrm{W}$. On discussing the heat capacity of metals, possible contributions of the conduction 
electrons must be considered. This important point needs to be discussed in more detail. It turns out that the well known linear-in-T electronic heat capacity is a typical low temperature phenomenon. At high temperatures, where the heat capacity of many metallic solids is a linear function of temperature as well, this contribution has nearly completely disappeared. This can be verified best for metals with a large coefficient $\gamma$ of the low temperature electronic heat capacity such as niobium with $\gamma=7.79 \mathrm{mJK}^{-2} \mathrm{~mole}^{-1}$ [19]. For niobium the slope of the linear high temperature heat capacity according to [5] is $4.23 \mathrm{mJK}^{-2} \mathrm{~mole}^{-1}$ only (see Fig. 2). Consequently, the electronic heat capacity must have strongly decreased with respect to the low temperature linear-in-T function. Change from increasing to decreasing temperature dependence is indicative of a crossover event in the conduction band. In other words there seem to be two alternative excitation spectra in the conduction band. One excitation spectrum must be attributed to bosons in the (spatially continuous) conduction band. This excitation spectrum is responsible for the universal linear-in-T electronic heat capacity at low temperatures. The other excitation spectrum is relevant (activated) at elevated temperatures and has a vanishing heat capacity compared to the low temperature linear-in-T function of the boson field. Only the second excitation spectrum conforms to the classical band theories of metals [20]. In the following data analyses it appears justified to neglect electronic contributions to the high temperature heat capacity of the metals.

Figure 1 shows high temperature heat capacity data of vanadium as a function of absolute temperature [5, 10]. Data of [10] labeled by curve \#1 (filled points) show perfect linear temperature dependence. The slope of this line $\left(10.75 \mathrm{mJK}^{-}\right.$ $\left.{ }^{2} \mathrm{~mole}^{-1}\right)$ is only slightly larger than the coefficient of the low temperature linear-in- $\mathrm{T}$ electronic heat capacity of $\gamma=9.26$ $\mathrm{mJK}^{-2} \mathrm{~mole}^{-1}$ [19]. Since linear-in-T high temperature heat capacities with similar slopes as for curve \#1 in Figure 1 are observed in insulators as well it can be concluded that the observed high temperature linear-in- $\mathrm{T}$ heat capacity of the metals is not of electronic origin.

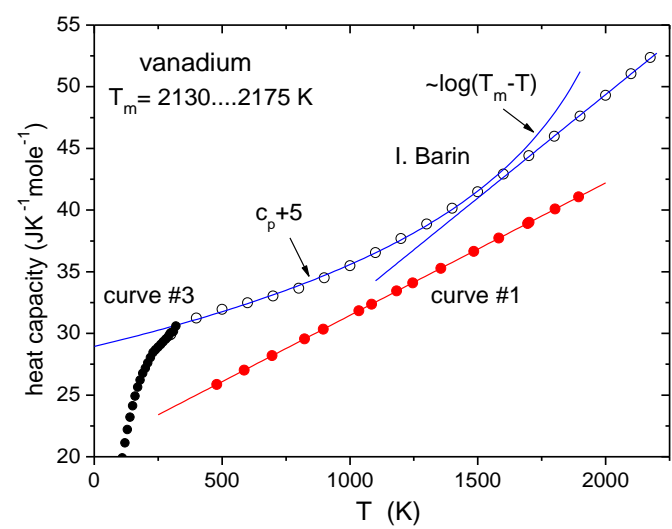

Figure 1. High temperature heat capacity of vanadium from two literature sources as a function of absolute temperature [5, 10]. While data of [10] labeled by curve \#1 exhibit perfect linear temperature dependence $(\alpha=1)$ data of [5] show crossover from logarithmic behavior $(\alpha=0)$ to linear behavior. For better clarity heat capacity data of [5] have been shifted upwards along ordinate axis by $5 \mathrm{JK}^{-1} \mathrm{~mole}^{-1}$.

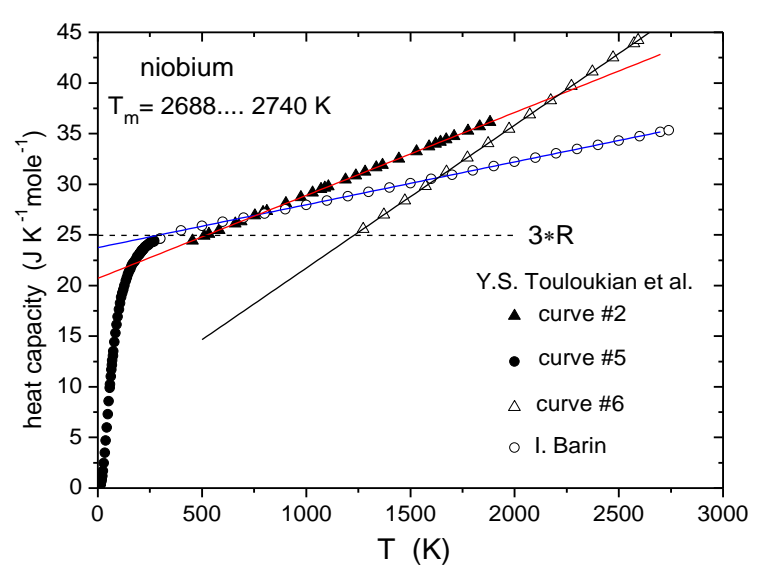

Figure 2. Temperature dependence of the heat capacity of niobium from two literature sources [5,10]. All data sets with heat capacities larger than D-P limit have linear temperature dependence but with different slopes and different absolute values at $T_{m}$. The different slopes indicate that the linear-in-T heat capacity cannot be due to conduction electrons. The slope of the curve labeled by Barin [5] of $4.23 \mathrm{mJK}^{-2}$ mole $^{-1}$ is less than the coefficient of the low temperature electronic heat capacity of $\gamma=7.79 \mathrm{mJK}^{-2}$ mole $^{-1}$ [19].

In the data of [5] (open points) crossover from low temperature logarithmic behavior $(\alpha=0)$ to linear behavior $(\alpha=1)$ is identified. For clarity these data have been shifted upwards along ordinate axis by $5 \mathrm{JK}^{-1} \mathrm{~mole}^{-1}$. Quantitatively similar but analytically different temperature dependence of the two data sets in Figure 1 shows that averaging over data sets of different authors is not reasonable.

Figure 2 shows a selection of heat capacity data of niobium all with linear temperature dependence $[5,10]$. The small statistical scatter of the data points clearly indicates that the different slopes and absolute constants of the three data sets must have a systematic origin.

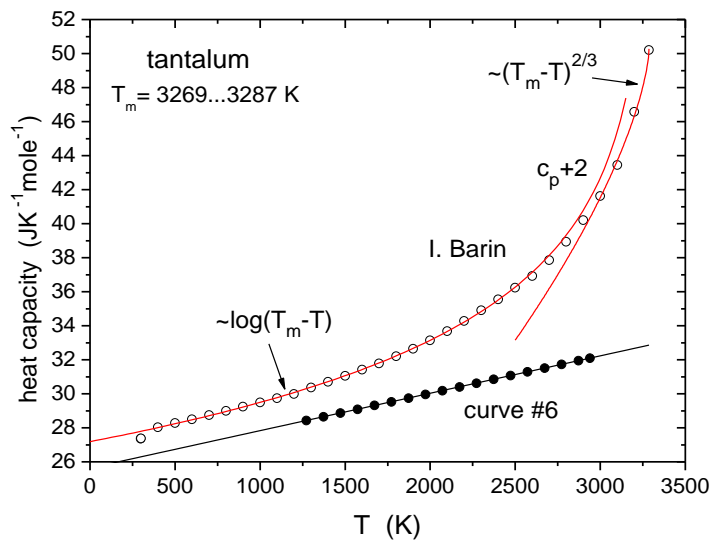

Figure 3. Heat capacity data of tantalum from two sources as a function of temperature $[5,10]$. The slope of curve \#6 of $2.2 \mathrm{mJK}^{-2}$ mole $^{-1}$ is less than the coefficient of the low temperature electronic heat capacity of $\gamma=5.9 \mathrm{mJK}^{-2}$ mole $^{-1}$ [19]. Data of [5] are well described over a large temperature range by logarithmic dependence $(\alpha=0)$. Asymptotically $\sim\left(T_{m}-T\right)^{2 / 3}$ gives reasonable description for $T \rightarrow T_{m}$. 


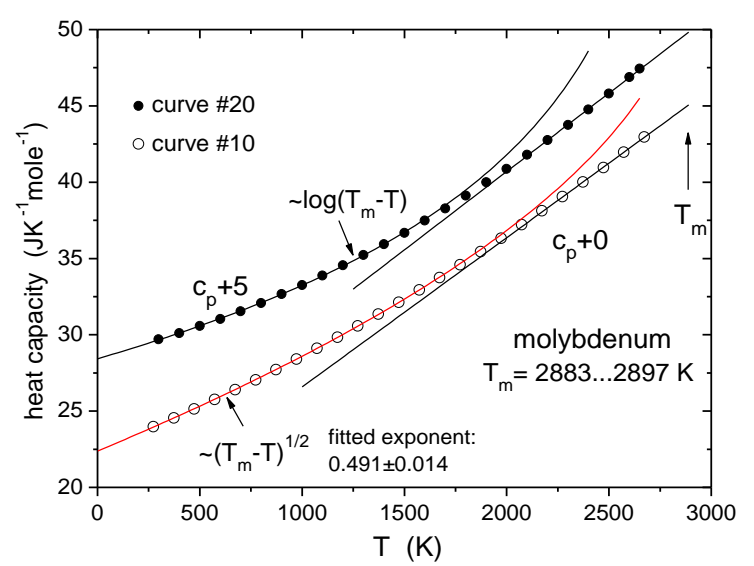

Figure 4. Two data sets of high temperature heat capacity of molybdenum as a function of absolute temperature [10]. Data of curve \#10 are well described by crossover from $\sim\left(T_{m}-T\right)^{1 / 2}$ function to linear function. In curve \#20 the crossover is from logarithmic to linear dependence. The latter data have been shifted upwards along ordinate axis by $5 \mathrm{JK}^{-1} \mathrm{~mole}^{-1}$.

A surprising behavior of curve \#2 and curve \#6 in Figure 2 is that these data do not approach the well established low temperature data with $\mathrm{c}_{\mathrm{p}}<3 R$ (curve \#5). This indicates that crossover to field dynamics is not necessarily at that temperature where the heat capacity has reached D-P limit but can be shifted to a much larger temperature. This could mean that there can be a gap between the two excitation spectra.

Figures 3-5 give quite analogous data analyses for the heat capacity of tantalum, molybdenum and tungsten. Note that for tungsten the heat capacities at $T_{m}$ are different by nearly $90 \%$ (Figure 5).

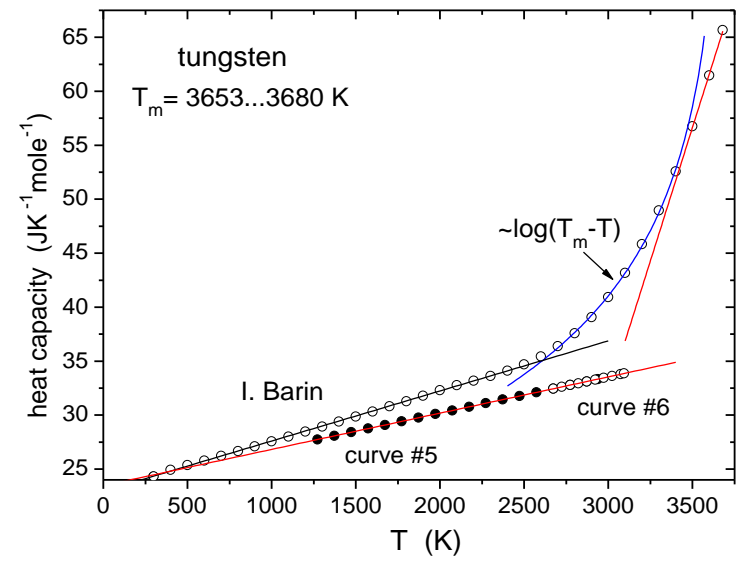

Figure 5. High temperature heat capacities of tungsten from two literature sources as a function of absolute temperature $[5,10]$. Data of curve \#5 and curve \#6 agree excellently and show linear temperature dependence. In the data of [5] crossover from linear to logarithmic function can be identified. Logarithmic divergence cannot be the asymptotic behavior. Asymptotic behavior seems to be linear again.

In this short communication only a limited number of experimental examples for universality in the critical heat capacity near melting point have been presented. In particular, no examples of the non asymptotic exponent of $\alpha=2$ (Al, curve \#4, Re curve \#2 [10]) and of the asymptotic exponent of $\alpha=1.5$ (Rh [5]) have been shown.

\section{Conclusions}

From an atomistic point of view all crystals of the same material should have identical properties and, as a consequence, should exhibit identical heat capacities. This expectation is satisfactorily fulfilled only for heat capacity values smaller than D-P limit of $3 R$. As a conclusion, atomistic lattice theory is correct for $c_{p}<3 R$ only where the dynamics is, in fact, determined by atomic vibrations. On the other hand it is long known that on approaching the melting point the reported heat capacities for the same material can deviate increasingly from each other by much more than the experimental error. In particular, for the same material the reported heat capacities at melting temperature can differ by more than $50 \%$. As a consequence, there must be an experimental parameter, not included in atomistic models, that has to be made responsible for those anomalies.

As we have shown, each individual data set can well be described by one, or a sequence of more, universal power functions of type $\sim\left(T_{m}-T\right)^{\alpha}$ with different (rational) values of $\alpha$ and different absolute constants. A strong indication for a non-atomistic critical dynamics is that the fitted critical power functions hold up to a large distance away from $T_{m}$. This is in contrast to the atomistic critical power functions that hold asymptotically at the critical point only. As a consequence, the observed high temperature heat capacity of solids is that of a boson field. This is nothing unusual since at low temperatures the heat capacity of all solids is also that of a boson field (the Debye boson field). The large mean free path of the field quanta near $T_{m}$ implies a new length scale to the dynamics [21]. Without interactions with the atomistic background the mean free path of the field bosons would be infinite. For a mean free path of larger than sample dimensions the sample acts as a cavity for the field bosons. The energy density of the field, and therefore the universality class of the heat capacity, that is the exponent $\alpha$, then depends on shape and surface quality of the sample. Definition of an intrinsic behavior appears hardly possible. When the critical dynamics above and below $T_{m}$ is independent of atomistic structures it follows that the ordering transition is executed by the boson field instead by inter-atomic interactions. Consequently, the actual value of the ordering temperature can depend somewhat on the shape of the sample. In fact, for the same material the reported melting temperatures are surprisingly different (see Figures 1 to 5). In RG theory it is customary to call the boson controlled critical temperature a stable fixed point (SFP) in order to distinguish it from the critical temperature predicted by atomistic models $[8,9]$.

Although RG theory was developed especially for the dynamics at magnetic ordering transitions its main conclusion that the critical dynamics in the vicinity of the magnetic ordering transition is that of a boson field seems to apply to many other types of order-disorder phase transitions as well such as the vapor-liquid transition [16,17]. However, in most cases the nature of the field quanta is unexplored. Development of realistic field theories of the critical dynamics at the various types of order-disorder phase transitions therefore is not yet possible.

Because of the limited space we have restricted discussion to a few monoatomic metallic elements. As investigations of many more materials, including insulating compounds [22] 
show, the here presented analyses of the high temperature heat capacity in terms of universal power functions of type $\sim\left(T_{m}-T\right)^{\alpha}$ works generally [22]. For instance, $c_{p}(T)$ data of [5] for $\mathrm{Al}_{2} \mathrm{O}_{3}$ are well described by crossover from $\sim\left(T_{m}-T\right)^{2}$ to asymptotic $\sim\left(T_{m}-T\right)$ function. We could identify asymptotic exponent values of $\alpha=2 / 3,1$ and $3 / 2$. As exponents of the non asymptotic power function $\alpha=0,1 / 2$ and 2 were identified. Considering that the sample shape can be assumed to be of importance on the actual exponent value it is surprising that a limited number of (rational) exponents occur only. This is typical for the stability of the universality classes. Non relevant parameters do not perturb the universality class. How the observed exponents can be correlated with shape and size of the sample is, however, unclear. Systematic heat capacity measurements on samples with different shape could clarify this question.

\section{References:}

[1] M.E. Fisher and A.N. Berker, "Scaling for first-order phase transitions in thermodynamic and finite systems," Physical Review B, 26, 2507-2513, 1982.

[2] H. Fenichel and B. Serin, "Low-temperature specific heat of solid neon and solid xenon," Physical Review 142, 490-495, 1966.

[3] H. Bilz and W. Kress, Phonon Dispersion Relations in Insulators Berlin: Springer, 1979.

[4] M. Born and K. Huang, Dynamical Theory of Crystal Lattices Oxford: Clarendon Press, 1956.

[5] I. Barin, Thermochemical Data of Pure Substances Weinheim: VCH, 1995.

[6] U. Köbler, "Boson fields in solid state physics," in Recent Developments in Bosons Research, I. Trambley (Ed.) New York: Nova Science Publishers, pp. 1-55, 2013.

[7] U. Köbler, "Towards a field theory of magnetism," in Recent Developments in Magnetism Research, K. Pace (Ed.), New York: Nova Science Publishers, pp. 1-66, 2013.

[8] K.G. Wilson and J. Kogut, "The Renormalization Group and the E Expansion," Physics Reports, 12C, 75-199, 1974.
[9] K.G. Wilson, "The Renormalization Group: Critical Phenomena and the Kondo Problem," Reviews of Modern Physics, 47, 773-840, 1975.

[10] Y.S. Touloukian and E.H. Buyco, Thermophysical Properties of Matter, Vol. 4, Specific heat of metallic elements and alloys New York: IFI/Plenum, 1970.

[11] A.J. Leadbetter, "Anharmonic effects in the thermodynamic properties of solids II. Analysis of data for lead and aluminium," Journal of Physics, C 1, 14891504, 1968.

[12] Ya. Kraftmakher, Lecture notes on equilibrium point defects and thermophysical properties of metals Singapore: World Scientific Publishing, p. 328, 2000.

[13] Ya. Kraftmakher, "Equilibrium vacancies and properties of metals at high temperatures," High Temperature Materials and Processes, 15, 171-178, 2011.

[14] R. Feder and A.S. Nowick, "Equilibrium vacancy concentration in pure $\mathrm{Pb}$ and dilute $\mathrm{Pb}-\mathrm{Tl}$ and $\mathrm{Pb}-\mathrm{In}$ alloys," Philosophical Magazin, 15, 805-812, 1967.

[15] U. Köbler and A. Hoser, "Magnetism of powder samples and of single crystals,"Journal of Magnetism and Magnetic Materials, 349, 88-94, 2014.

[16] P. Heller, "Experimental investigations of critical phenomena," Reports on Progress in Physics, 30, 731826, 1967.

[17] R.K. Pathria, Statistical Mechanics, $2^{\text {nd }}$ Ed. Oxford: Butterworth-Heinemann, 1996.

[18] U. Köbler and A. Hoser, "Magnetic interaction by exchange of field bosons," Acta Physica Polonica, A121, 1176-1178, 2012.

[19] C. Kittel, Introduction to Solid State Physics, $8^{\text {th }}$ Ed. New York: John Wiley\&Sons Inc. p.146, 2005.

[20] A. Sommerfeld and H. Bethe, Elektronentheorie der Metalle Berlin: Springer, 1967.

[21] U. Köbler and A. Hoser, "Single domain behavior of bulk $\mathrm{RbMnF}_{3}$," Journal of Magnetism and Magnetic Materials, 325, 87-93, 2013.

[22] Y.S. Touloukian and E.H. Buyco, Thermophysical Properties of Matter, Vol. 5, Specific heat of nonmetallic solids, New $\quad$ York: IFI/Plenum, 1970. 